EPJ Web of Conferences 41, 06004 (2013)

DOI: $10.1051 /$ epjconf/20134106004

(C) Owned by the authors, published by EDP Sciences, 2013

\title{
Ultrafast structural and vibrational dynamics of the hydration shell around DNA
}

\author{
T. Elsaesser, Ł. Szyc, and M. Yang
}

Max-Born-Institut für Nichtlineare Optik und Kurzzeitspektroskopie, 12489 Berlin, Germany

\begin{abstract}
Two-dimensional infrared spectroscopy in the frequency range of $\mathrm{OH}$ - and $\mathrm{NH}$ stretch excitations serves for a direct mapping of hydration dynamics around DNA. A moderate slowing down of structural dynamics and resonant $\mathrm{OH}$ stretch energy transfer is observed in the DNA water shell compared to bulk water.
\end{abstract}

The aqueous environment of biomolecules, the hydration shell, plays a key role for their macromolecular structure and their functional properties. While hydration phenomena have been studied in quite some detail under stationary conditions and in the time range accessible by NMR, there is very limited insight into ultrafast dynamics of water shells on the time scale of molecular motions and vibrational relaxation. A first approach of femtosecond spectroscopy is based on introducing chromophores into the biomolecular structure and measuring the transient red-shift of fluorescence or stimulated emission which is introduced by the solvation of the electronic dipole [1]. Apart from being structurally invasive, this method has difficulties to discern the response due to polar groups of the biomolecule from structural dynamics of the water shell. As a result, the interpretation of such data has remained controversial [1,2].

Here, we introduce femtosecond two-dimensional (2D) infrared spectroscopy to study the structural and vibrational dynamics of water shells around biomolecules in the electronic ground state. Selecting particular vibrations as probes, molecular couplings and ultrafast processes in the biomolecule and the water shell can be separated. As a prototype system, DNA oligomers containing 23 alternating adenine-thymine base pairs in Watson-Crick geometry are studied at different hydration levels $[3,4]$. We separate the $\mathrm{NH}$ stretch excitations of the base pairs and their mutual couplings from $\mathrm{OH}$ stretch excitations of the water shell which undergo a pronounced spectral diffusion on a time scale of 500 fs. A quantitative analysis of such $\mathrm{OH}$ stretch dynamics gives evidence of a slowing-down of structural fluctuations and resonant vibrational energy transfer in the water shell compared to bulk water.

DNA films of a thickness of approximately $10 \mu \mathrm{m}$ were prepared on $500 \mathrm{~nm}$ thick $\mathrm{Si}_{3} \mathrm{~N}_{4}$ substrates [3]. The film samples were integrated in a humidity cell in which a well-defined relative humidity (RH) was maintained by connecting the cell to a reservoir containing different agents for $\mathrm{RH}$ levels ranging from $0 \% \mathrm{RH}(\sim 2$ water molecules per base pair) up to $92 \% \mathrm{RH}$ (more than 20 water molecules per base pair). The $2 \mathrm{D}$ spectra were derived from heterodyne-detected 3-pulse photon echoes recorded with mid-infrared pulses of $7 \mu \mathrm{J}$ energy and a duration of 50-70 fs. The photon-echo experiments were performed in a boxcar geometry and two phase-locked pulse pairs were generated with the help of diffractive optics.

The 2D spectra recorded at $0 \%$ RH (Fig. 1) are dominated by NH stretch excitations of the base pairs. There are two strong diagonal peaks, the upper one at $3350 \mathrm{~cm}^{-1}$ due to the asymmetric $\mathrm{NH}_{2}$

This is an Open Access article distributed under the terms of the Creative Commons Attribution License 2.0, which permits unrestricted use, distribution, and reproduction in any medium, provided the original work is properly cited. 


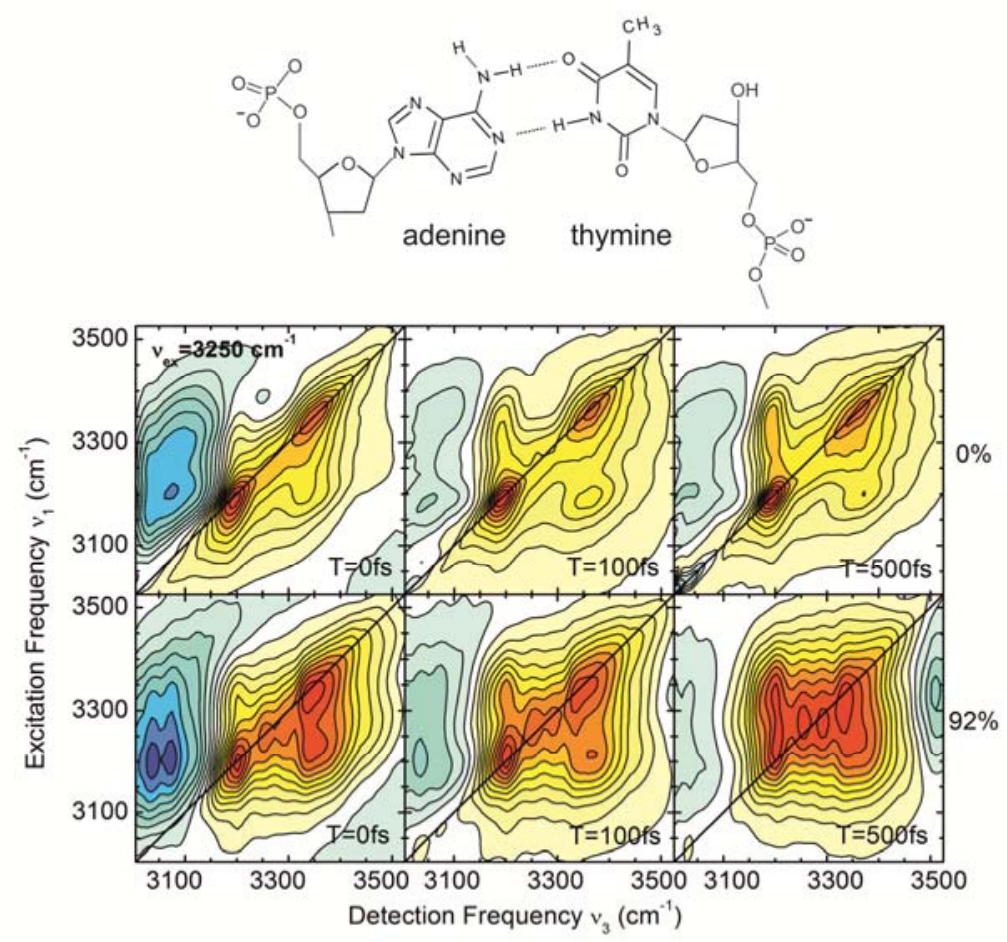

Fig. 1. Top: Molecular structure of an adenine-thymine base pair in Watson-Crick geometry. Bottom: 2D infrared spectra measured for different population times $\mathrm{T}$ at a humidity level of $0 \%$ $\mathrm{RH}$ (upper row) and $92 \% \mathrm{RH}$ (lower row). The femtosecond pulses were centered at $3250 \mathrm{~cm}^{-1}$. The 2D signals above $v_{3} \approx 3150 \mathrm{~cm}^{-1}$ are due to $\mathrm{NH}$ or $\mathrm{OH}$ stretch $\mathrm{v}=0$ to 1 excitations, signals below to $\mathrm{v}=1$ to 2 transitions. The amplitude change between neighboring contour lines is $10 \%$. The $0 \% \mathrm{RH}$ spectra are dominated by $\mathrm{NH}$ stretch excitations of the base pairs. The $92 \% \mathrm{RH}$ spectra represent a superposition of $\mathrm{NH}$ stretch and $\mathrm{OH}$ stretch excitations of the water shell.

stretch mode of adenine and the lower one at $3200 \mathrm{~cm}^{-1}$ representing a superposition of the symmetric $\mathrm{NH}_{2}$ stretch mode of adenine and the $\mathrm{NH}$ stretch vibration of thymine. The cross peaks reveal the coupling between the different NH stretch modes. As a function of population time T, the intensity of the cross peak at $\left(v_{1}, v_{3}\right)=(3350,3200) \mathrm{cm}^{-1}$ increases relative to the diagonal peaks, due to a downhill energy transfer from the asymmetric $\mathrm{NH}_{2}$ stretch vibration of adenine to the $\mathrm{NH}$ stretch mode of thymine. All diagonal and cross peaks show minor spectral diffusion as a function of T.

For fully hydrated DNA $(92 \% \mathrm{RH})$, the 2D spectra display a superposition of the NH stretch peaks and the $\mathrm{OH}$ stretch contribution of the water shell (Fig. 1). The latter shows a pronounced reshaping from a diagonal to an essentially round line shape on a time scale of $500 \mathrm{fs}$, much slower than the sub-100 fs spectral diffusion in bulk water [5]. The presence of the water shell leads to a moderate broadening of the $\mathrm{NH}$ stretch peaks only, pointing to a limited influence of water fluctuations on the NH stretch lineshapes.

For a more detailed comparison of the hydration shell dynamics with bulk water, we measured 2D spectra with pulses centered at $3400 \mathrm{~cm}^{-1}$ (Fig. 2a). Here, the OH stretch contributions strongly dominate and the reshaping due to spectral diffusion can be quantified by analyzing the slopes of center lines (thick solid lines in Fig. 2a) as a function of population time T. The reshaping from the initial inhomogeneously broadened elliptic line shape into a more homogeneous round shape leads to $a$ decrease of the center line slopes within the first 500 fs (open circles in Fig. 2b). Fig. $2 b$ also shows frequency-time correlation functions of bulk water calculated from molecular dynamics simulations which include resonant energy transfer between $\mathrm{OH}$ stretching oscillators (solid line) or neglect this mechanism (dashed line) [6]. 

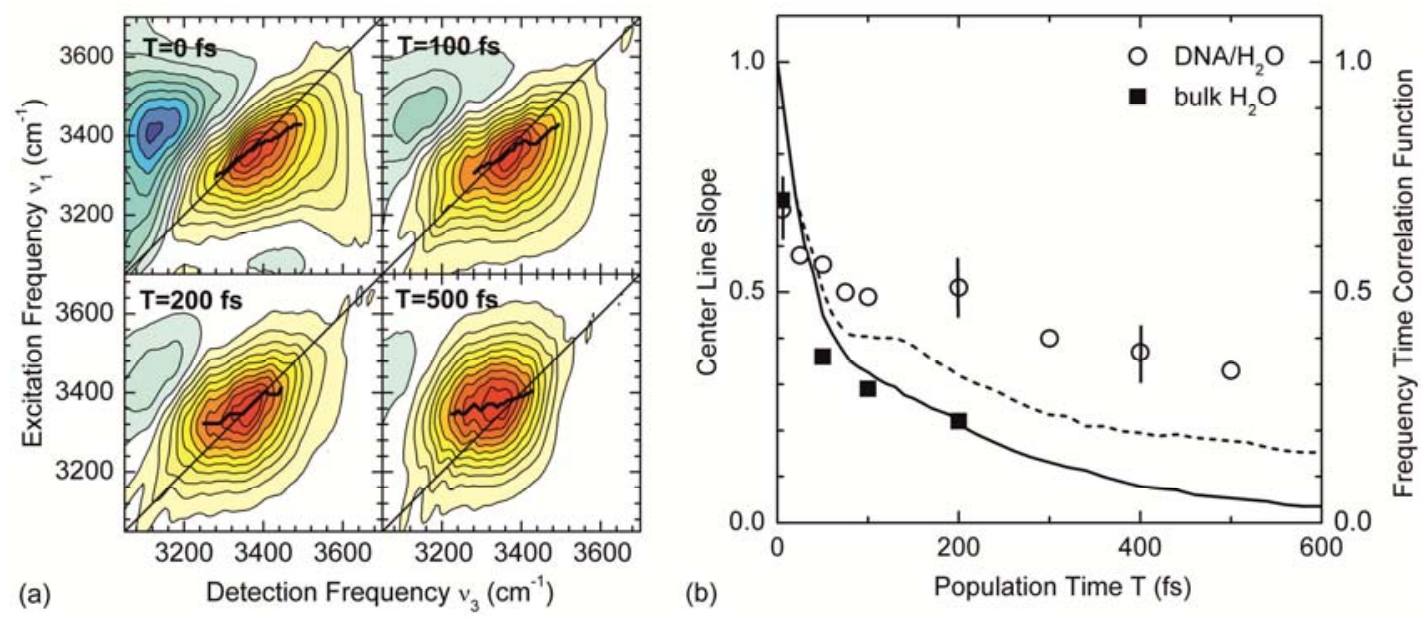

Fig. 2. (a) $2 \mathrm{D}$ infrared spectra measured for different population times $\mathrm{T}$ at a humidity level of $92 \%$ $\mathrm{RH}$ using femtosecond pulses centered at $3400 \mathrm{~cm}^{-1}$. The spectra are dominated by $\mathrm{OH}$ stretch $\mathrm{v}=0$ to 1 excitations of the water shell. Thick solid lines: center lines derived from cuts along the excitation frequency axis. (b) Center line slopes derived from 2D spectra of the DNA hydration shell (open circles) and of bulk water (solid squares) as a function of population time T. The solid and the dashed line are calculated frequency-time correlation functions of bulk water from Ref. [6].

Compared to such calculations and experimental results (squares, Ref. [5]) for bulk water, the DNA hydration shell displays a smaller amplitude of the fast sub-100 fs decay which is mainly due to librational motions, and a moderately slower time evolution between 100 and $500 \mathrm{fs}$. We attribute this behavior to the more rigid structure of the hydration shell, in particular of the first water layer interacting with DNA, and to a smaller rate of resonant $\mathrm{OH}$ stretch energy transfer at the reduced water concentration of $\mathrm{c} \approx 10 \mathrm{M}$.

Vibrational relaxation of $\mathrm{NH}$ and $\mathrm{OH}$ stretch excitations and energy dissipation in the water shell were studied in an independent series of experiments [4,7]. In both the water shell and in bulk water, a vibrationally hot ground state which is characterized by an enhanced fraction of structurally distorted and/or broken hydrogen bonds is formed on a 1 to 2 ps time scale.

1. Z. Dongping, P.S. Kumar, A.H. Zewail, Chem. Phys. Lett. 503, 1 (2011)

2. B. Halle, L. Nilsson, J. Phys. Chem. B 113, 8210 (2009)

3. M. Yang, Ł. Szyc, T. Elsaesser, J. Phys. Chem. B 115, 1262 (2011)

4. M. Yang, Ł. Szyc, T. Elsaesser, J. Phys. Chem. B 115, 13093 (2011)

5. D. Kraemer, M.L. Cowan, A. Paarmann, N. Huse, E.T.J. Nibbering, T. Elsaesser, R.J.D. Miller, Proc. Natl. Acad. Sci. USA 105, 437 (2008).

6. T.L.C. Jansen, B.M. Auer, M. Yang, J.L. Skinner, J. Chem. Phys. 132, 224503 (2010)

7. Ł. Szyc, M. Yang, T. Elsaesser, J. Phys. Chem. B 114, 7951 (2010) 